\title{
Decoherence and Relaxation Time of Magnetopolaron in the Presence of Three Dimensional Impurity Under Strong Parabolic Potential
}

\author{
Bernard Donfack*, Ghislain Tsopgue Tedondje, Tetchoka Manemo Cedric, \\ Cornesse Drugile Guimapi Ngoufack, Alain Jervé Fotue
}

Mesoscopic and Multilayers Structures Laboratory, Department of Physics, Faculty of Science, University of Dschang, Dschang, Cameroon

Email address:

bdonfack94@yahoo.fr (B. Donfack)

${ }^{*}$ Corresponding author

\section{To cite this article:}

Bernard Donfack, Ghislain Tsopgue Tedondje, Tetchoka Manemo Cedric, Cornesse Drugile Guimapi Ngoufack, Alain Jervé Fotue. Decoherence and Relaxation Time of Magnetopolaron in the Presence of Three Dimensional Impurity Under Strong Parabolic Potential. American Journal of Modern Physics. Vol. 10, No. 5, 2021, pp. 101-110. doi: 10.11648/j.ajmp.20211005.11

Received: August 22, 2021; Accepted: September 3, 2021; Published: October 21, 2021

\begin{abstract}
In order to protect coherence of quantum states and reduce the impact of environment on quantum information, we investigate decoherence and relaxation time of magnetopolaron in the presence of three dimensional impurity under strong parabolic potential. The first states energies have been evaluated using the Lee Low Pine transformation and Pekar-type variational method. Parameters such as: decoherence time, transition frequency, spontaneous emission, Shannon entropy, relaxation time and probability density, have been evaluated. It has been seen that the impurity and electron-phonon coupling constant have a considerable effect on formation, protection of quantum qubit and quantum transport. The information exchange measured by the rate of Shannon entropy, has a great dependence on impurity and with its interaction with electrons. The relaxation time $\tau_{r}$ exhibits increasing behavior as a function of, $\alpha, \beta$, and $\omega_{c}$. The electron-phonon coupling constant, impurity and cyclotron frequency are useful parameters to prevent decoherence phenomena. This study paves the way to prolong quantum effect in nanostructure and favor the realization of the future quantum computer.
\end{abstract}

Keywords: Magnetopolaron, Strong Parabolic Potential, Three Dimensional Impurity, Decoherence, Relaxation Time

\section{Introduction}

Quantum information is nowadays an intermediate agent between quantum mechanics and information science. In this way, it can combine the both. The particularity of this quantum information is that the carrier of quantum information can be found in two different states and the fact that it can be easy, faster solve and compute spontaneously calculations. Quantum information contains a different unit of storage called qubit and this qubit refers to two superposed quantum states $|\downarrow\rangle$ and $|\uparrow\rangle$ which satisfy the condition of orthogonality and normalization. $|q u b i t\rangle=a|\downarrow\rangle+b|\uparrow\rangle ; a$ and $b$ represent respectively probabilities that particles can be found in the following quantum states $|\downarrow\rangle$ and $|\uparrow\rangle$ with $|a|^{2}+|b|^{2}=1$. As there is no perfect system in physics generally, in quantum information, transport of information and quantum modeling are influenced by the effect of environment. Due to the presence of impurity and external environment, the instability of coherence of quantum state is observed (decreases the coherence of quantum state) which is called qubit decoherence. Then an increase of decoherence causes the disappearance of superiority of quantum computation. Therefore, the quantum decoherence becomes a new challenge for the researchers about how to protect the quantum information from its surrounding? Recently, many works have been done in the same direction. Some have studied the suppressing decoherence by applying external field and even spin-orbit coupling [1-7] and obtained some 
important analysis. A non-neglected number of investigations have been done on the loss of coherence state and how to build a long decoherence time system? [8-11] which allows controlling such system. Since the human needs keep increasing, the new technology with quantum computer is requested to be more useful and faster than classical computer and need to be realized. To this end, many methods and devices have been proposed but the most feasible approaches are nanostructures generally. As magnetopolaron is contained in quantum system, it is obvious that it can be stable in one hand and unstable on the other hand in quantum system. But what we need in quantum system is a coherent state to perform an efficiency of quantum devices in general and quantum computer particularly. Since it is required that a qubit should be isolated from external environment [12, 13]. From the last past decade, the electronic structure, electronic optical of low dimensional system have became the main subject of questioning in condensed matter physics. In this way, many works both experimental [1417] and theoretical [18-21] have been done on quantum system. Using a Pekar variation method, $\mathrm{Mu}$ et al [22] investigated the Eigenenergies and the corresponding Eigenfunction of the ground state and excited state energy of an electron strongly coupled to electron-phonon in $\mathrm{RbCl}$ quantum pseudo dot taking as impurity hydrogen at the centre. They obtained after analysis that: first the probability density of the electron was an oscillator function in quantum pseudodot (QPD) with a certain oscillating period. Second the asymmetrical potential in the direction of $\mathrm{RbCl} \mathrm{QPD}$ was responsible to the appearance of double peak on the electron probability density. And that an oscillation period decreases witha columbic impurity potential. Because of that the impurity state has great effect in physical properties of low dimensional system, the impurity state have been studied in theoretical and experimental procedures [23-25]. Many authors [26-32] have performed considerably the binding energy of a hydrogen impurity in a quantum well and they found that the cross-sectional of quantum wire can have a great effect on the binding energy. B. Donfack et al studied the cumulative effects of temperature, magnetic field and spin orbit interaction on the binding energy of magnetopolaron in $\mathrm{RbCl}$ quantum well [33], their numerical results show that, the spin orbit interaction affect considerably the GSBE of magnetopolaron and the transition behavior at threshold values of the temperature as well as for cyclotron frequency is due to the effect of SOI. Liang et al [34] have studied the eigenenergies and eigenfunction of the ground state and first excited state of strong coupled polaron in asymmetric quantum dot via variational method of Pekar-type. Fotué et al [35] investigated decoherence of polaron in an asymmetric quantum dot qubit under electromagnetic field and their analysis show that the density matrix of qubit decay with time. And for different coupling strengths, confinement length and dispersion coefficient, the coherence term is a decreasing function with time. Sun et al [36] investigated the decoherence problem of polaron in triangular quantum dot and LI [37] has investigated the dependence of polaron on temperature in triangular quantum dot. Choose a proper confinement potential function for the threedimensional confinement of electron in quantum system is really important. However, in many works, the single parametric parabolic potential is an ideal model that is too simplified and it's useful to better study and clearly interprets our results. Recently, some authors [38, 39] chose the two parametric asymmetric Gaussian potential to describe the motion of electrons 2D quantum structures. However, decoherence and relaxation time of threedimensional impurity magnetopolaron in the presence of strong parabolic potential need to be investigated then, the purpose of this paper is based on that direction by solving analytically the Schrödinger equation to obtain the complete energy spectrum in the presence of strong parabolic potential. The present paper is organized as follow: in section 2, theoretical model and formulation, where the Schrödinger equation is analytically solved and the ground state and first excited state energy are obtained using the LLPT and Pekar-type variational method. In section 3, we have numerical results and discussion of parameters obtained in previous section, in the next section, numerical results and discussions are presented. The last section conclude our analysis.

\section{Theoritical Model and Formulation}

We suppose an electron moving and confined in the threedimensional system, assuming that it interacts with impurity and bulk LO phonon in the medium. The strong parabolic potential is chosen to confine electrons along the $\mathrm{x}-\mathrm{y}$ plane. The magnetic field is applied with the vector potential $A=\frac{B}{2}(-y, x, 0)$ directed in the z-direction interacting with phonon. The Hamiltonian of such system can be written as [37]:

$$
\begin{gathered}
H=H_{e}+V(\rho)+V_{c}(r)+H_{p h}+H_{e-p h} \\
H_{e}=\frac{1}{2 m}\left(p+\frac{e A}{c}\right)^{2} \\
V(\rho)=\frac{m \omega^{2}}{2}\left(x^{2}+y^{2}\right) \\
V_{c}(r)=-\frac{e^{2}}{4 \pi \varepsilon_{\infty} r} \\
H_{p h}=\sum_{q} \hbar \omega_{L O} a_{q}^{+} a_{q} \\
\text { and } H_{e-p h}=\sum_{q}\left[V_{q} a_{q} e^{i q r}+V_{q}^{*} a_{q}^{+} e^{-i q r}\right]
\end{gathered}
$$

represent respectively the kinetic energy of an electron, the strong parabolic potential which confine electrons, the coulomb energy considered like impurity, the phonon energy and electron-phonon interaction energy. Replacing those terms in equation (1), the hamiltonian becomes: 


$$
H=\frac{1}{2 m}\left(p+\frac{e A}{c}\right)^{2}+\frac{m \omega^{2}}{2}\left(x^{2}+y^{2}\right)+\sum_{q} \hbar \omega_{L O} a_{q}^{+} a_{q}+\sum_{q}\left[V_{q} a_{q} e^{i q r}+V_{q}^{*} a_{q}^{+} e^{-i q r}\right]-\frac{e^{2}}{4 \pi \varepsilon_{\infty} r}
$$

Where:

$$
\begin{gathered}
V_{q}=i\left(\frac{\hbar \omega_{q}}{q}\right)\left(\frac{\hbar}{2 m \omega_{q}}\right)^{\frac{1}{4}}\left(\frac{4 \pi \alpha}{v}\right)^{\frac{1}{2}} \\
\alpha=\left(\frac{e^{2}}{2 \hbar \omega_{q}}\right)\left(\frac{2 m \omega_{q}}{\hbar}\right)^{\frac{1}{2}}\left(\frac{1}{\varepsilon_{\infty}}-\frac{1}{\varepsilon_{0}}\right)
\end{gathered}
$$

and the fourier transformation of the last term of hamiltonian is given by:

$$
-\frac{\beta}{r}=-\sum_{q} \frac{4 \pi \beta}{V q^{2}} \exp (-i q r)
$$

In order to evaluate the magnetopolaron energy, let's use the LLPT and Pekar-type variational method. In this way, the chosen wave function for the ground state and first excited is

$$
\begin{gathered}
\left|\phi_{0}\right\rangle=\left(\frac{\varepsilon_{0}}{\pi}\right)^{\frac{1}{2}}\left(\frac{\vartheta_{0}}{\pi}\right)^{\frac{1}{4}} \exp \left(-\frac{\varepsilon_{0}}{2} \rho^{2}\right) \exp \left(-\frac{\vartheta_{0}}{2} z^{2}\right)\left|0_{p h}\right\rangle \\
\left|\phi_{1}\right\rangle=\left(\frac{\varepsilon_{1}}{\pi}\right)^{\frac{1}{2}}\left(\frac{\vartheta_{1}}{\pi}\right)^{\frac{1}{4}} \rho \exp \left(-\frac{\varepsilon_{0}}{2} \rho^{2}\right) \exp \left(-\frac{\vartheta_{0}}{2} z^{2}\right) \exp ( \pm i \phi)\left|0_{p h}\right\rangle
\end{gathered}
$$

Where $\varepsilon_{0}, \varepsilon_{1}, \vartheta_{0}$ and $\vartheta_{1}$ represent the variational parameters, $\left|\phi_{0}\right\rangle$ and $\left|\phi_{1}\right\rangle$ should satisfy the following normalization conditions: $\langle 0 \mid 0\rangle=1$ and $\langle 0 \mid 1\rangle=0$.

Using the LLPT and Pekar-type variational method,

$$
E_{0}=\frac{\hbar^{2}}{2 m}\left(\varepsilon_{0}+\frac{\vartheta_{0}}{2}\right)+\frac{m}{2 \varepsilon_{0}}\left(\frac{\omega_{c}^{2}}{2}+\omega^{2}\right)+\left(1-\frac{\varepsilon_{0}}{\vartheta_{0}}\right)^{-\frac{1}{2}} \arcsin \left(1-\frac{\varepsilon_{0}}{\vartheta_{0}}\right)^{\frac{1}{2}}\left[-2 \alpha\left(\frac{\hbar^{3} \omega_{L O}}{\pi}\right)^{-\frac{1}{2}}-\frac{e^{2}}{\varepsilon_{\infty} \pi^{2}}\left(4 \pi \varepsilon_{0}\right)^{\frac{1}{2}}\right]
$$

and

$$
E_{1}=\frac{\hbar^{2}}{2 m}\left(2 \varepsilon_{1}-3 \vartheta_{1}\right)+\frac{m}{\varepsilon_{1}}\left(\omega_{c}^{2}+\omega^{2}\right)+\left(1-\frac{\varepsilon_{1}}{\vartheta_{1}}\right)^{-\frac{1}{2}} \arcsin \left(1-\frac{\varepsilon_{1}}{\vartheta_{1}}\right)^{\frac{1}{2}}\left[8 \alpha\left(\frac{\hbar^{2} \varepsilon_{1} \omega_{L O}}{m \pi}\right)^{\frac{1}{2}}-\frac{e^{2}}{\varepsilon_{\infty} \pi^{2}}\left(4 \pi \varepsilon_{0}\right)\right]
$$

Knowing the ground state $E_{0}$ and first excited $E_{1}$ energy, the time evolution of the different state of the electrons can be given as:

$$
\psi_{01}(x, y, z, t)=\frac{1}{\sqrt{2}}\left|\phi_{0}(\rho)\right\rangle \exp \left(-i \frac{E_{0}}{\hbar} t\right)+\frac{1}{\sqrt{2}}\left|\phi_{1}(\rho)\right\rangle \exp \left(-i \frac{E_{1}}{\hbar} t\right)
$$

Using the following formular, 


$$
Q(x, y, z, t)=\left|\psi_{01}(x, y, z, t)\right|^{2}
$$

The probability density is written as:

$$
Q(x, y, z, t)=\frac{1}{2}\left[\left|\phi_{0}(\rho)\right|^{2}+\left|\phi_{1}(\rho)\right|^{2}+\phi_{0}^{*}(\rho) \phi_{1}(\rho) \exp \left(-\omega_{01} t\right)+\phi_{0}(\rho) \phi_{1}^{*}(\rho) \exp \left(-\omega_{01} t\right)\right]
$$

Where $\omega_{01}$ represents the energy gap defined as:

$$
\omega_{01}=E_{1}-E_{0}
$$

And then $\frac{\omega_{01}}{\hbar}=\frac{E_{1}-E_{0}}{\hbar}$ represents the transition frequency between the ground state and first excited. We can define the oscillation period here as an inverse of transition frequency and its expression is:

$$
T=\frac{\hbar}{E_{1}-E_{0}}
$$

Taking into account the great effect of external environment on the superposition states of electron, introducing the impurity and strong parabolic potential, the spontaneous emission rate $\tau^{-1}$ of the LO phonon is defined as [38]:

$$
\begin{gathered}
\tau^{-1}=\frac{e^{2} \Delta E}{2 \pi c \varepsilon_{0} \hbar^{2} \varsigma}\left|\left\langle\psi_{0}|\vec{r}| \psi_{1}\right\rangle\right|^{2} \\
\text { with } \varsigma=\frac{\left(\omega_{L O}-\omega\right)}{c q^{2}}
\end{gathered}
$$

Where $\tau$ is defined as an inverse of spontaneous emission and allows to measuredecoherence time of a qubit.

Since the loss of energy can be measured by the rate of entropy, lets evaluate the Shannon entropy using the following formula:

$$
S(t)=\int d x d y d z\left|\psi_{\phi_{o} / \phi_{1}}(t, x, y, z)\right|^{2} \ln \left|\psi_{\phi_{o} / \phi_{1}}(t, x, y, z)\right|^{2}
$$

The relaxation time by simple definition is the time taken by particle to leave from the excited state to the ground state (from disturbed state to undisturbed state). This physical quantity can destroy the superposed quantum state depending to the values taken; relaxation time can be evaluated using the formula below.

$$
\tau_{r}=\tau_{0} \exp \left(\frac{\Delta E}{K T}\right)=\tau_{0} \exp \left(\frac{E_{1}-E_{0}}{K T}\right)
$$

$K$ and $T$ are respectively the Boltzmann constant and temperature.

\section{Numerical Results and Discussion}

This part is devoted to numerical results and discussions considering some approximations as:

$$
m=0.36 m_{0}, \hbar \omega_{q}=36.4 ; K_{B}=1
$$

Figures 1 and 2 display respectively the ground state and first excited state energy as function of impurity for different values of magnetic field. In the both figures, we observe an increase of $E_{0}$ and $E_{1}$ with an increase of impurity. Knowing that an increase of impurity increases an interaction between it and electrons, this presence of impurity makes electrons strongly bound and consequently increases their energy and even their binding energy. The curves spacemen on the ground state energy allows concluding that this impurity acts more at this state than on the excited state. Similar to the result found in [41], the impurity has a great effect compared to others external parameters on energy state and entropy.

Figures 3 and 4 show the variation of ground state and first excited state energy as function of electron-phonon coupling constant $\alpha$ for different values of cyclotron frequency $\omega_{c}$ : we observe on those figures an increase of the ground state and first excited energy with a magnetic field and the coupling constant, this linear increasing of energy with coupling constant is due to the interaction between an electron and phonon, as magnetic field and coupling constant are both the confining parameter, an increase observed on those different energy states is obvious. This increasing of energies with electron-phonon coupling constant means that electrons and phonons strongly interact. Thus we have less polaron in the excited state and consequently leading to the less energy compared to the ground state.

Figures 5 and 6 display respectively the variation of probability density $Q(x, y, z, t)$ as function of impurity and coupling constant $\alpha$. It is observed in figure 5 that, probability density $Q(x, y, z, t)$ varies with impurity $\beta$. From here, we see that it increases with an increase of impurity. It is observe that the dependence of probability density is an isotropic, this is why an amplitude and period are not constant. It is because of the greater columbic attraction between electron and impurity. Knowing that the impurity can be localized in the system, its position (centre or position else) can equally affect the probability density in the system. In figure 6 , we have the dependence of probability density $Q(x, y, z, t)$ on $\alpha$, we observe here that $Q(x, y, z, t)$ is a periodic function of $\alpha$, with the same amplitude and this for $\alpha \in[0-5]$, as phonon is usually considered as vibration of medium, an amplitude of this vibration is constant but electron-phonon interaction is not, that why probability density has a periodic variation with electron-phonon coupling constant. Then we can conclude that: from those figures, $Q(x, y, z, t)$ is a non-periodic function with impurity compared to the one with electron-phonon 
coupling constant. This observation can be justify by saying that the impurity position in the system is variable and then, it can completely affect the parameters of the system such as energy, probability density while the electron-phonon coupling constant has a constant effect at the whole point of the system.

Figures 7 and 8 show respectively the variation of the oscillation period $T$ as function of the impurity and electronphonon coupling constant $\alpha$. From those figures, we can observe that $T$ decreases with an increase of coupling constant $\alpha$. Since $T=\hbar / \Delta E$, this is because the energy state $E_{0}$ and $E_{1}$ all are increase functions with a coupling constant $\alpha$ and impurity then causes a decrease of oscillation period since it's inversely proportional to the energy gap $\Delta E$. From the previous analysis, we can conclude that the impurity $\beta$ and coupling constant $\alpha$ have a great effect on life time of the qubit and the most important is the storage of information.

Figures 9 and 10 display the spontaneous emission of LO phonon $\tau^{-1}$ as function of impurity and cyclotron frequency $\omega_{c}$. It can be observed on those figures that $\tau^{-1}$ decrease with an increase of electron-phonon coupling constant $\alpha$ but keep increasing with $\beta$ and $\omega_{c}$ for some values greater than 6 . Which means that decoherence time $\tau$ increases with an increase of $\alpha$ and decreases with the impurity and magnetic field. Therefore in order to reduce the impact of environment on formation of qubit, storage and quantum transport from the ground state to excited state, the impurity should be adjusted and localized. The variation of spontaneous emission (inverse of decoherence time $\tau$ ) with coupling constant, shows a decrease of $\tau^{-1}$ with $\alpha$, this means that decoherence time $\tau$ is a decrease function of $\alpha$. From the previous analysis, we can conclude that the electron-phonon coupling constant $\alpha$ has a considerable effect and from the stronger coupling constant $\alpha$, resulting in the smaller the spontaneous emission of LO phonon then the longer decoherence time can be produced. The present result can inspire the using of phonon effect to prepare a quantum system qubit with good coherence, and also make semiconductor materials in intermediate and strong coupling constant.

Figures 11 and 12 show the variation of transition frequency $\omega$ respectively as function of impurity and electron-phonon coupling constant $\alpha$. On the both figures, we have an increase of transition frequency with an increase of impurity $\beta$ and $\alpha$. This increase of $\omega$ with those parameters can be due to the presence of a strong parabolic potential. From this increase of $\omega$, we conclude that the energy gap $\Delta E$ decreases with the increase of impurity and coupling constant $\alpha$. The main reason is also that $E_{0} \succ E_{1}$ in the case of magnetopolaron and polaron [42]. Knowing that the energy gap decreases with the e-phonon coupling constant $\alpha$, and that the number of phonon at the excited state is always less than that of the ground state [43], then the effect of $\alpha$ on the excited state energy is less comparedto the ground state which causes the transition frequency spacing with an increase of $\alpha$.

In the presence of impurity, the energy and binding energy decrease [41] and electron has more space to move without interaction and therefore, the system disorder increases. But for a very small cross-sectional area, we have an increases of entropy in the presence of impurity because there is more interaction between electrons and impurity. Since in the presence of impurity, we observe an increase of energy [41] we can now come out from the present analysis that electrons interact more and more in the presence of impurity. From this analysis, it is observed that impurity is a good factor to control the rate of effect of an environment on our system (decoherence) since the effect of impurity might increase coherence of superposed state (quantum qubit).

Figure 13 show respectively the dependence of Shannon entropy $S$ on impurity $\beta, \alpha$ and time $t$. On figure 13a, we observe that Shannon entropy doesn't have the same peak value with an increase of impurity. This can be due to the interaction between this impurity and electrons; $S$ is higher when interaction is stronger and the nature of interaction depends on the position of impurity in the system. Then, from this analysis, the peak value of the Shannon entropy here depends on the position of impurity and its interaction with electrons. Figure 13b displays the variation of Shannon entropy with e-phonon coupling constant $\alpha$. It is observed the Shannon entropy $S$ has the same peak value with an increase of $\alpha$, which means the rate of order or disorder created from $\alpha$ is the same in the whole point of system. Contrary from the analysis done by S. C Kenfack et al [44] in which they found that the variation of Shannon entropy with time has the same amplitude, we found on figure $13 \mathrm{c}$ that, for some values of time [1-2.3] and [3.4-4.5] in $n s$, we the highest peak values of entropy in the system and for $t \in[2.1-3.1]$ in $n s$, we have more ordered states and this because of the smallest values of entropy. This difference can be due to the presence of impurity in our system. As we know, an increase of entropy can be responsible of the loss of an information and coherent state in quantum system, then we can solve a problem of decoherence by minimizing the rate of entropy in quantum system.

Figures 14 and 15 show the variation of relaxation time $\tau_{r}$ with impurity $\beta$ for different values of e-phonon coupling constant $\alpha$ and cyclotron frequency $\omega_{c}$ on the one hand and the variation of relaxation time $\tau_{r}$ with an e-phonon coupling constant $\alpha$ for different values of impurity $\beta$ and cyclotron frequency $\omega_{c}$ on the other hand. We observe on the both figures an increase of relaxation time impurity $\tau_{r}$ with those parameters ( $\alpha, \beta$, and $\omega_{c}$ ). From this analysis, we can conclude that: contrary to decoherence time, a long relaxation time is not suitable to the construction of quantum qubit because as longer $\tau_{r}$ will be long, more particle will stay in the separated state. Moreover, a long relaxation time can creates a shot decoherence time. In order to build a quantum qubit, a long decoherence time and shot relaxation time are required, and this throughout the adjusting of the parameters $(\alpha, \beta$, and $\omega_{c}$ ) of system and the choice of potential. 


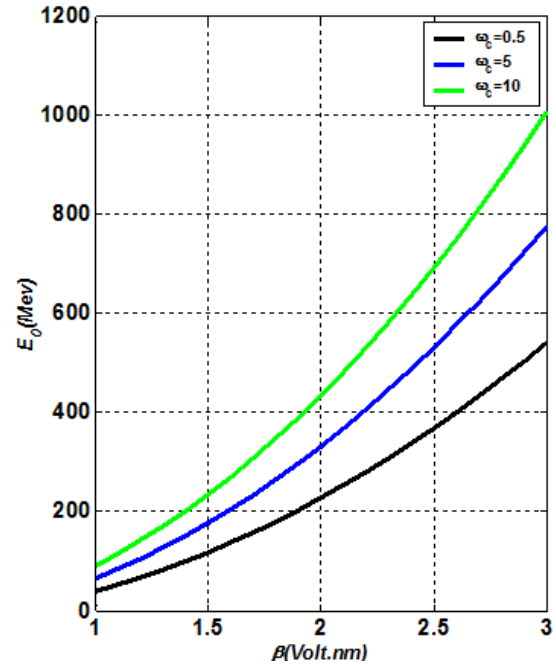

Figure 1. Variation of ground state energy as function of impurity for different values of magnetic field.

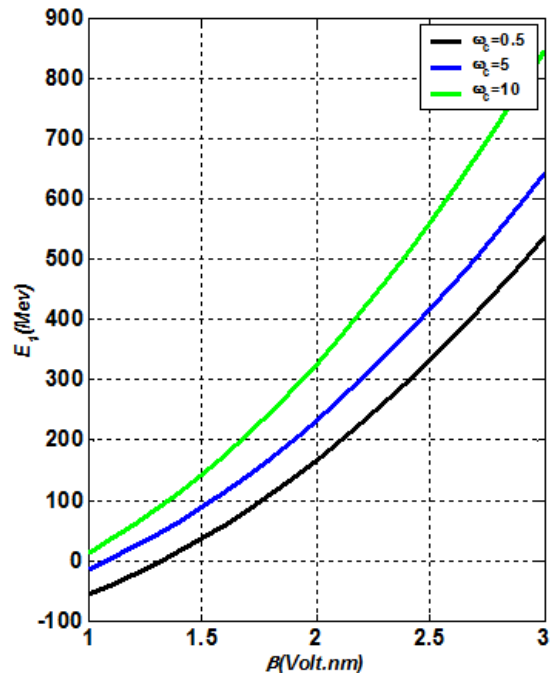

Figure 2. Variation of first excited state energy as function of impurity for different values of magnetic field.

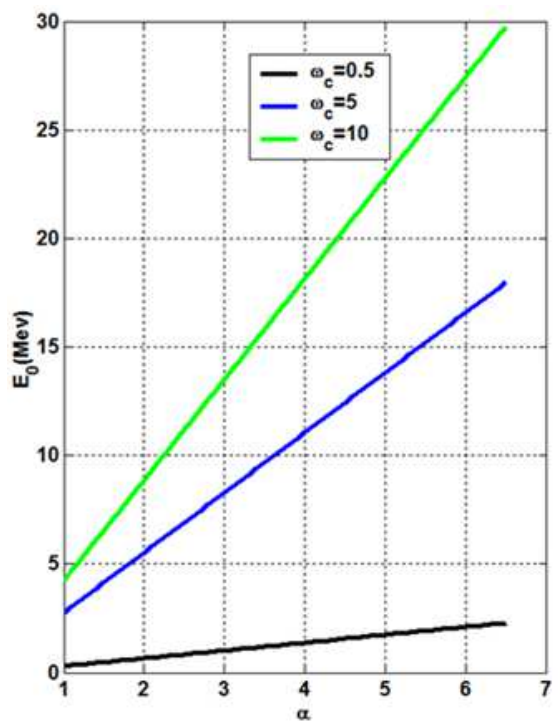

Figure 3. Variation of ground state energy as function of impurity for different values of electron-phonon coupling constant $\alpha$.

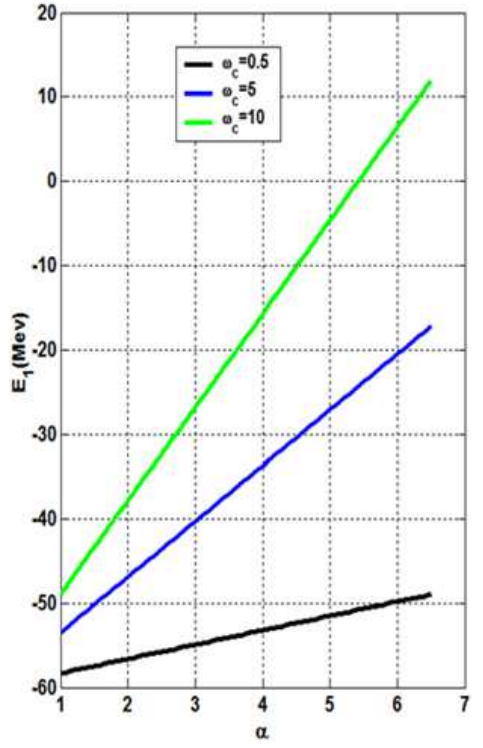

Figure 4. Variation of first excited state energy as function of impurity for different values of electron-phonon coupling constant $\alpha$

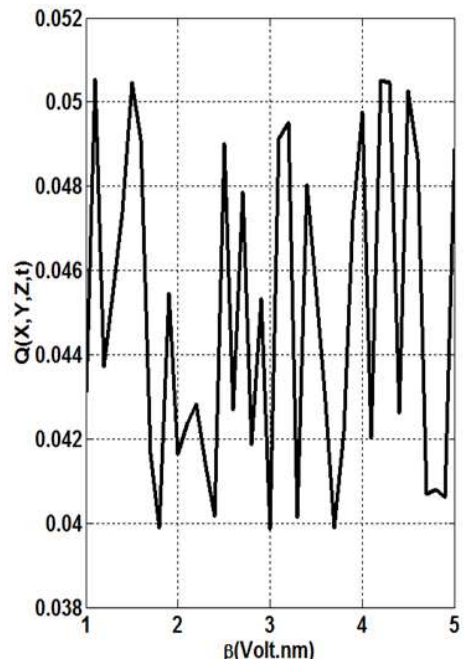

Figure 5. Variation of probability density as function of impurity.

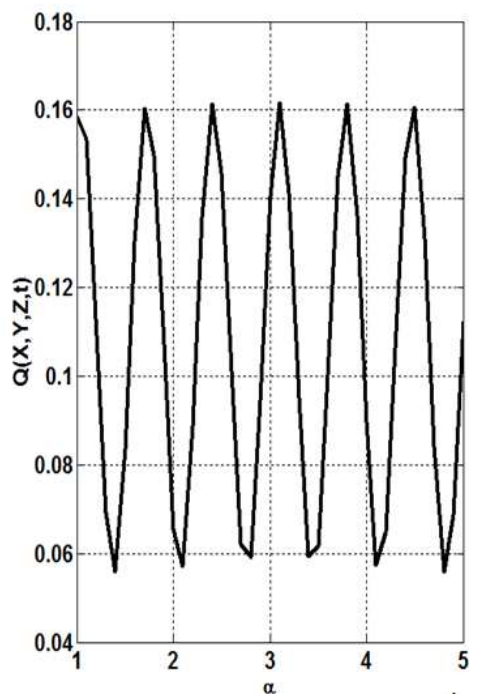

Figure 6. Variation of probability density as functionof electron-phonon coupling constant $\alpha$. 


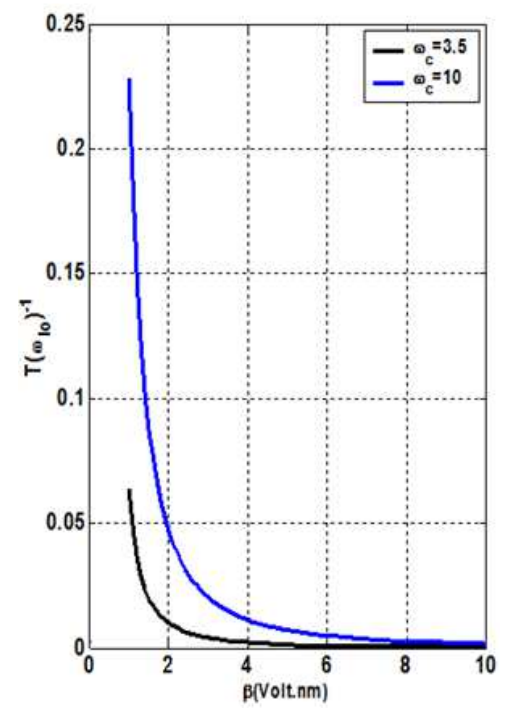

Figure 7. The oscillation period $T$ as function of impurity $\beta$ for different values of magnetic frequency $\omega_{c}$

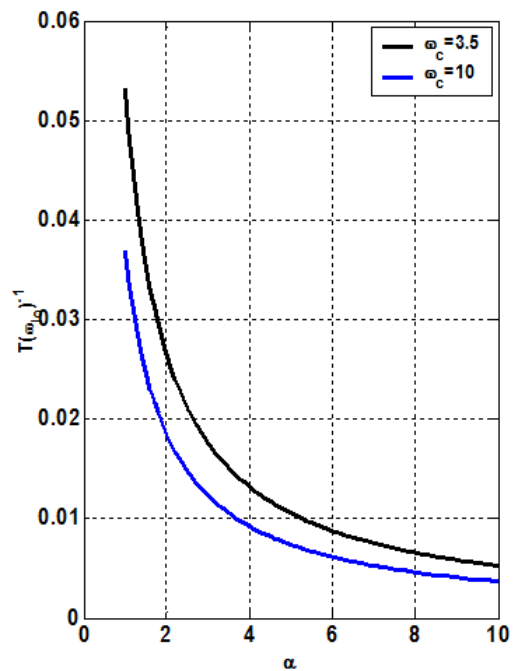

Figure 8. The oscillation period $T$ as function of electron-phonon coupling constant $\alpha$.

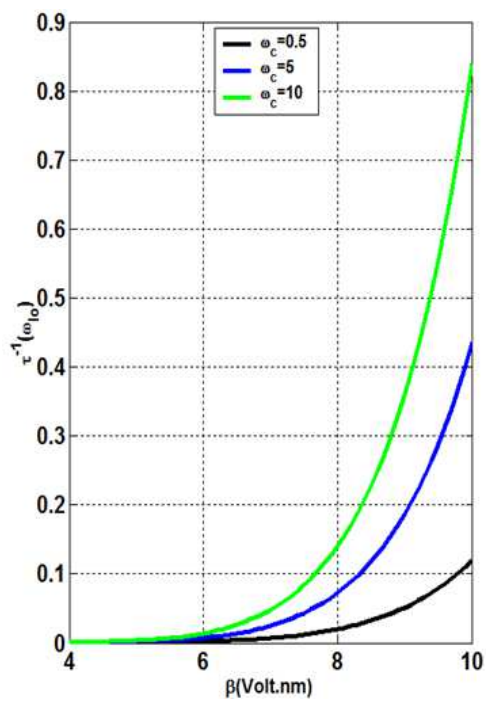

Figure 9. The spontaneous emission rate $\tau^{-1}$ as function of impurity $\beta$ for different values of cyclotron frequency $\omega_{c}$.

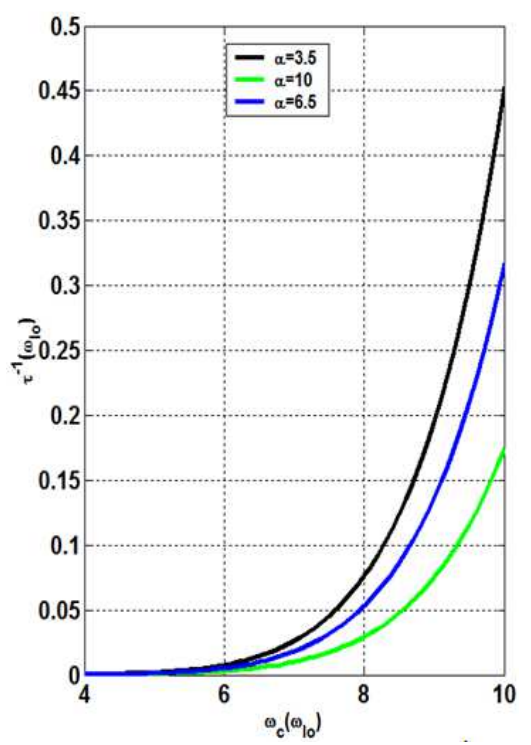

Figure 10. The spontaneous emission rate $\tau^{-1}$ as function of cyclotron frequency $\omega_{c}$ for different values of electron-phonon coupling constant $\alpha$.

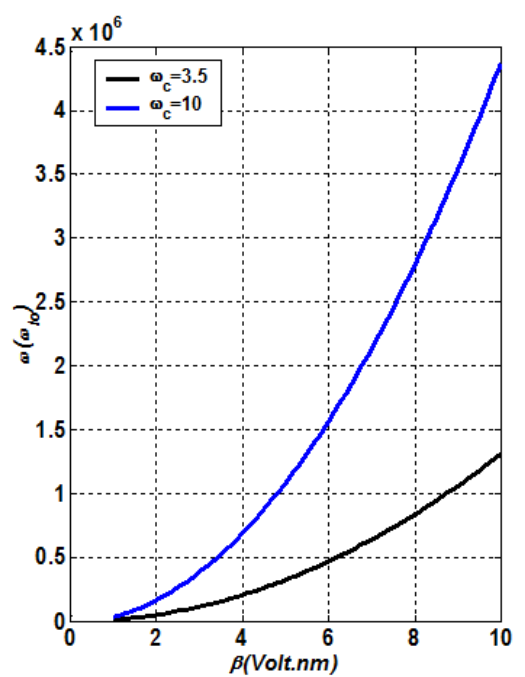

Figure 11. The transition frequency $\omega$ as function of impurity $\beta$ for different values of cyclotron frequency $\omega_{c}$.

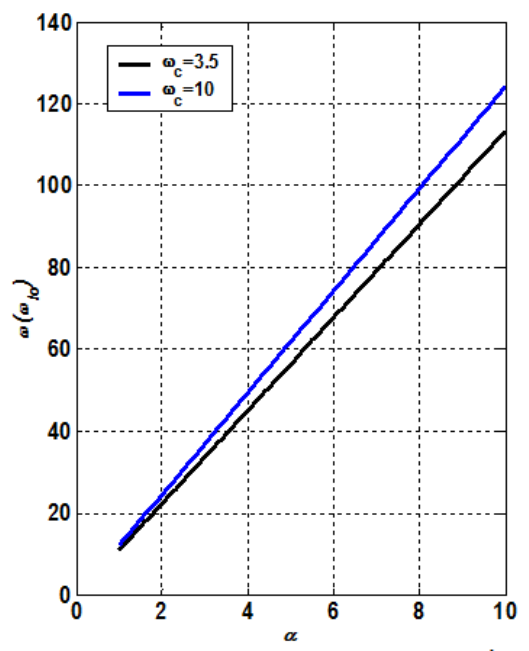

Figure 12. The transition frequency $\omega$ as function of electron-coupling constant $\alpha$ for different values of cyclotron frequency $\omega_{c}$. 


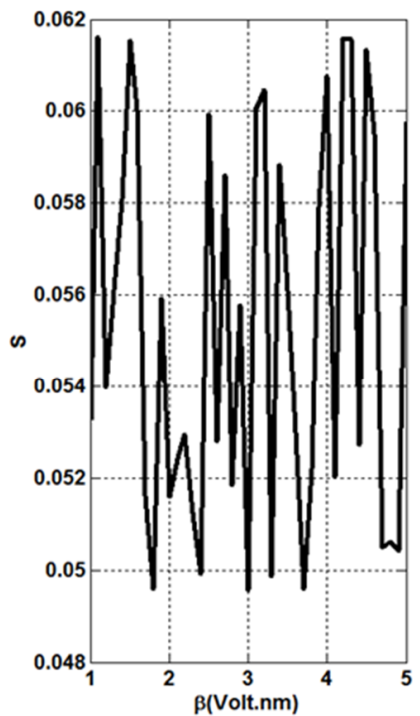

a

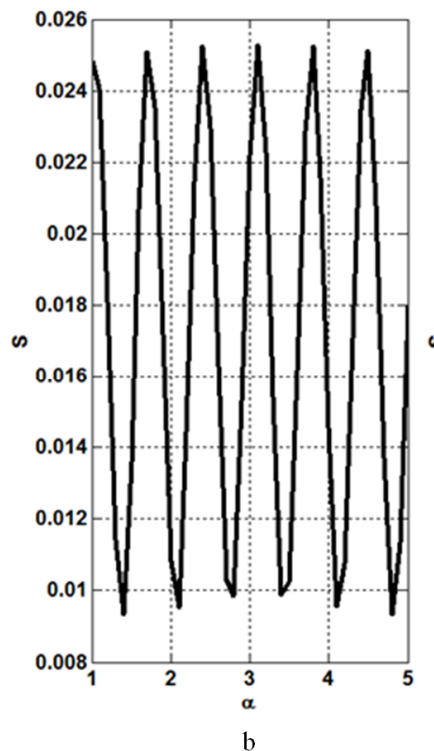

b

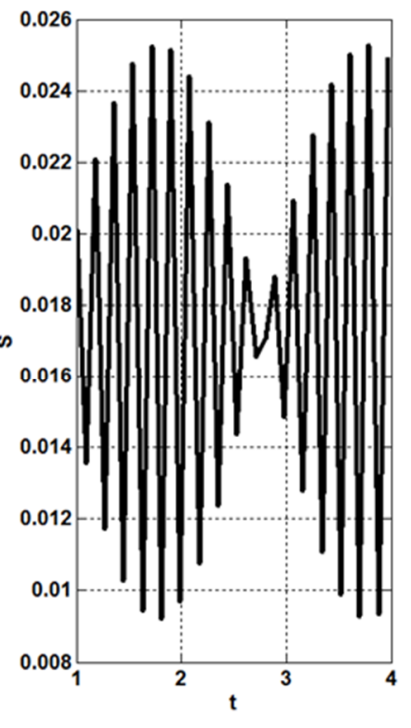

c

Figure 13. Present respectively Shannon entropy as function of impurity $\beta$, e-phonon coupling constant $\alpha$ and time $t$.

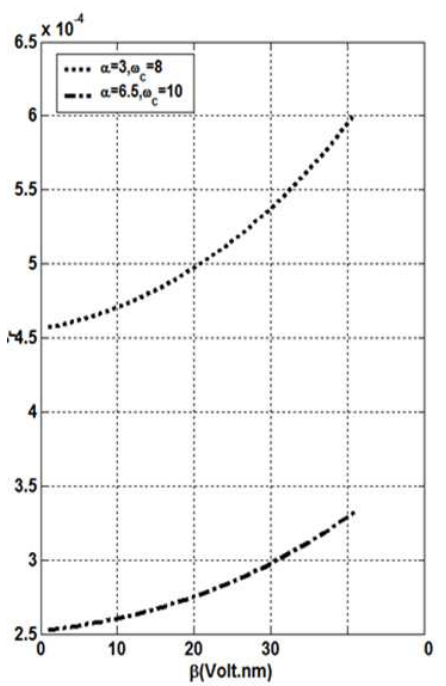

Figure 14. Relaxation time as function of impurity $\beta$ for different values of e-phonon coupling constant $\alpha$ cyclotron frequency $\omega_{c}$.

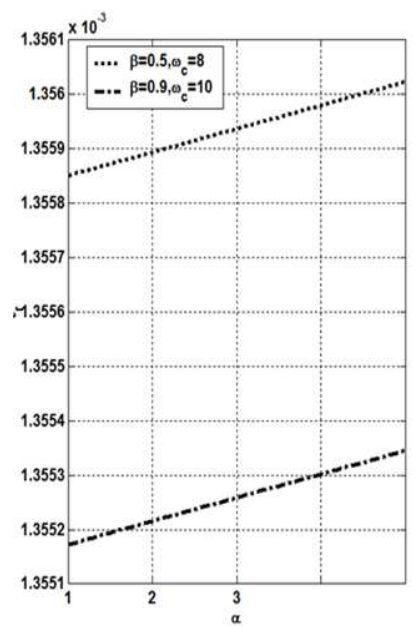

Figure 15. Relaxation time $\tau_{r}$ as function of e-phonon coupling constant $\alpha$ for different values ofimpurity $\beta$ and cyclotron frequency $\omega_{c}$.

\section{Conclusion}

We derived the ground state and first excited energy of the three dimensional impurity magnetopolaron in the presence of a strong parabolic potential using LLPT and Pekar-type variational method, we have equally evaluated some important parameters such as probability density, transition frequency, spontaneous emission, decoherence time, Shannon entropy, relaxation time and oscillation period. From our analysis, in the presence of impurity, the ground state and excited state energy are increasing functions of impurity and electrons are more bounded due to the strong interaction between that impurity and electron. We observe that variation of Shannon entropy depends on the position of impurity in the system and also with its interaction with electrons, an increase of that entropy can create the loss of information (decoherence) from the ground state to excited state. We observe an increase of relaxation time $\tau_{r}$ with those parameters ( $\alpha, \beta$, and $\omega_{c}$ ). Contrary to decoherence time, a long relaxation time is not suitable to the construction of quantum qubit because as longer $\tau_{r}$ will be long, more particle will stay in the separated states. We have also seen that the probability density increases with an increase of impurity but compared to electron-phonon coupling constant, we have a non-periodic function and non-continue probability density with impurity because its effect depends on its position in the system. Which can in term of decoherence allows to have a good coherent states. We also conclude that decoherence time decreases with an increase of impurity and magnetic field. In order to reduce the impact of environment (decoherence on formation of quantum qubit, storage and quantum transport from ground state to excited state) the impurity should be adjusted and localized in the system. As we said before, in numerical results and discussion, the variation of spontaneous emission with electron-phonon coupling constant allows to conclude that 
the decoherence time $\tau$ is an increase function of $\alpha$. With a qubit constructed, the effect of impurity an e-phonon coupling constant $\alpha$ on transition frequency from one state to another is great because we have an increase of transition frequency with the both parameters. Then due to the effect of impurity, coupling constant and magnetic field on the problem of protection of quantum qubit formed from environment, we can conclude that those parameters are prominent candidates to enhance the formation and duration of quantum qubit particularly and help for the construction of quantum computers.

\section{Conflict of Interest}

The authors declare that they have no conflict of interest.

\section{References}

[1] Li, Shu-Shen, Jian-Bai Xia, Jin-Long Liu, Fu-Hua Yang, ZhiChuan Niu, Song-Lin Feng, and Hou-Zhi Zheng. "InAs/GaAs single-electron quantum dot qubit." Journal of Applied physics 90, no. 12 (2001): 6151-6155.

[2] Petta, J. R., Johnson, A. C., Taylor, J. M., Laird, E. A., Yacoby, A., Lukin, M. D.,... \& Gossard, A. C. (2005). Coherent manipulation of coupled electron spins in semiconductor quantum dots. Science, 309 (5744), 21802184 .

[3] Varwig, S., René, A., Greilich, A., Yakovlev, D. R., Reuter, D., Wieck, A. D., \& Bayer, M. (2013). Temperature dependence of hole spin coherence in (In, Ga) As quantum dots measured by mode-locking and echo techniques. Physical Review B, 87 (11), 115307.

[4] Sun, Y., \& Xiao, J. L. (2019). The magnetic field effect on the coherence time of qubit in $\mathrm{RbCl}$ crystal quantum pseudodot. Optical and Quantum Electronics, 51 (4), 110.

[5] Xiao, J. L. (2019). The effect of Coulomb impurity potential on the coherence time of $\mathrm{RbCl}$ quantum pseudodot qubit. Journal of Low Temperature Physics, 195 (5-6), 442-449.

[6] Ma, X. J., \& Xiao, J. L. (2018). Temperature effects of the electron probability density on quantum pseudodot qubit. Optical and Quantum Electronics, 50 (3), 144.

[7] Xiao, J. L. (2018). The effects of hydrogen-like impurity and temperature on state energies and transition frequency of strong-coupling bound polaron in an asymmetric Gaussian potential quantum well. Journal of Low Temperature Physics, 192 (1-2), 41-47.

[8] J. P. Barnes, \& W. S. Warrenc Decoherence and programmable quantum computationlu201d, Phys. Rev. A 60, 6, 4363-4374 (1999); quant-ph/9902084.61.

[9] Machnikowski, P. (2008). Coherent control and decoherence of charge states in quantum dots. In Condensed Matter Physics In The Prime Of The 21st Century: Phenomena, Materials, Ideas, Methods (pp. 119-158).

[10] Yu, T., \& Eberly, J. H. (2006). Quantum open system theory: bipartite aspects. Physical review letters, 97 (14), 140403.

[11] Jordan, S. P., Farhi, E., \& Shor, P. W. (2006). Error-correcting codes for adiabatic quantum computation. Physical Review A, 74 (5), 052322.

[12] Xiao, J. L. (2016). Effects of temperature and hydrogen-like impurity on the coherence time of $\mathrm{RbCl}$ parabolic quantum dot qubit. Superlattices and Microstructures, 90, 308-312.

[13] Barkhouse, D. A. R., Debnath, R., Kramer, I. J., Zhitomirsky, D., Pattantyus-Abraham, A. G., Levina, L.,...\& Sargent, E. H. (2011). Depleted bulk heterojunction colloidal quantum dot photovoltaics. Advanced Materials, 23 (28), 3134-3138.

[14] Shalom, M., Albero, J., Tachan, Z., Martínez-Ferrero, E., Zaban, A., \& Palomares, E. (2010). Quantum dot- dye bilayer-sensitized solar cells: breaking the limits imposed by the low absorbance of dye monolayers. The journal of physical chemistry letters, 1 (7), 11341138.

[15] Maune, B. M., Borselli, M. G., Huang, B., Ladd, T. D., Deelman, P. W., Holabird, K. S.,...\& Sokolich, M. (2012). Coherent singlet-triplet oscillations in a silicon-based double quantum dot. Nature, 481 (7381), 344-347.

[16] Hansom, J., Schulte, C. H., Le Gall, C., Matthiesen, C., Clarke, E., Hugues, M.,... \& Atatüre, M. (2014). Environmentassisted quantum control of a solid-state spin via coherent dark states. Nature Physics, 10 (10), 725-730.

[17] Li, S. S., \& Xia, J. B. (2007). Binding energy of a hydrogenic donor impurity in a rectangular parallelepiped-shaped quantum dot: quantum confinement and Stark effects. Journal of applied physics, 101 (9), 093716.

[18] Datta, N. K., \& Ghosh, M. (2011). Excitations in doped quantum dot induced by randomly fluctuating magnetic field: influence of impurity. The European Physical Journal B, 80 (1), 95-103.

[19] Wang, C. S., \& Xiao, J. L. (2012). Transition frequency of weakcoupling impurity bound magnetopolaron in an anisotropic quantum dot. Modern Physics Letters B, 26 (01), 1150003.

[20] Xiao, J. L. (2014). Influences of temperature and impurity on excited state of bound polaron in the parabolic quantum dots. Superlattices and Microstructures, 70, 39-45.

[21] Ma, X. J., Qi, B., \& Xiao, J. L. (2015). Coulomb impurity potential $\mathrm{RbCl}$ quantum pseudodot qubit. Journal of Low Temperature Physics, 180 (3-4), 315-320.

[22] Sharkey, J. J., Yoo, C. K., Peter, A. J.: Magnetic field induced diamagnetic susceptibility of a hydrogenic donor in a GaN/AlGaN quantum dot. Superlatt. Microstruct. 48, 248-255 (2010).

[23] Bryant, G. W.: Hydrogenic impurity states in quantum-well wires: shape effects. Phys. Rev. B 31, 7812-7818 (1985).

[24] Bastard, G.: Superlattice band structure in the envelopefunction approximation. Phys. Rev. B 24, 5693-5697 (1981).

[25] Leonora, J. M., \& Peter, A. J. (2010). The effect of magnetic field on acceptor impurity in a diluted magnetic quantum well system. Solid state communications, 150 (1-2), 30-35.

[26] Crnjanski, J. V., Gvozdic, D. M.: Self-consistent treatment of Vgroove quantum wire band structure in non parabolic approximation. Serb. J. Electr. Eng. 1, 69-77 (2004).

[27] Chuu, D. S., Hsiao, C. M., Mei, W. N.: Hydrogenic impurity states in quantum dots and quantum wires. Phys. Rev. B 46, 3898-3905 (1992). 
[28] Zhu, J. L. (1989). Exact solutions for hydrogenic donor states in a spherically rectangular quantum well. Physical Review B, 39 (12), 8780.

[29] Jayam, S. G., \& Navaneethakrishnan, K. (2003). Effects of electric field and hydrostatic pressure on donor binding energies in a spherical quantum dot. Solid State Communications, 126 (12), 681-685.

[30] Khordad, R., Bahramiyan, H.: Study of impurity position effect in pyramid and cone like quantum dots. Eur. Phys. J. Appl. Phys. 67, 20402-20408 (2014).

[31] Oyoko, H. O., Duque, C. A., Montenegro, N. P.: Uniaxial stress dependence of the binding energy of shallow donor impurities in $\mathrm{GaAs}(\mathrm{Ga}, \mathrm{Al})$ Asquantumdots. J. Appl. Phys. 90, 819-823 (2001).

[32] DONFACK, B., FOTIO, F., FOTUE, A., \& Fai, L. C. (2020). Cumulative effects of temperature, magnetic field and Spin orbit Interaction (SOI) on the properties of magnetopolaron in $\mathrm{RbCl}$ quantum well. Chinese Journal of Physics.

[33] Liang, Z. H., Qi, B., \& Xiao, J. L. (2015). The effect of hydrogen-like impurity on $\mathrm{RbCl}$ asymmetric quantum dot qubit. Indian Journal of Physics, 89 (12), 1247-1250.

[34] Fotue, A. J., Kenfack, S. C., Issofa, N., Tiotsop, M., Djemmo, M. P. T., Wirngo, A. V.,...\& Fai, L. C. (2015). Decoherence of polaron in asymmetric quantum dot qubit under an electromagnetic field. Am J Mod Phys, 4 (3), 138-148.

[35] Sun, J. K., Li, H. J., \& Xiao, J. L. (2009). Decoherence of the Triangular Bound Potential Quantum Dot Qubit. Modern Physics Letters B, 23 (27), 3273-3279.

[36] Li, Z. X. (2012). Temperature-dependence of strong-coupled bound polaron in a triangular potential quantum dot. Modern Physics Letters B, 26 (03), 1150015.
[37] Xie, W. F. (2004). Condensed Matter: Electronic Structure, Electrical, Magnetic, and Optical Properties-Two Interacting Electrons in a Spherical Gaussian Confining Potential Quantum Well. Communications in Theoretical Physics, 42 (1), 151-154.

[38] Adamowski, J., Sobkowicz, M., Szafran, B., \& Bednarek, S. (2000). Electron pair in a Gaussian confining potential. Physical Review B, 62 (7), 4234.

[39] Cahay, M., \& Bandyopadhyay, S. (2017). Problem Solving in Quantum Mechanics: From Basics to Real-world Applications for Materials Scientists, Applied Physicists, and Devices Engineers. John Wiley \& Sons.

[40] Khordad, R., Sedehi, H. R., \& Bahramiyan, H. (2018). Effects of impurity and crosssectional shape on entropy of quantum wires. Journal of Computational Electronics, 17 (2), 551-561.

[41] Bai, X. F., Xin, W., \& Yin, H. W. (2017). Electromagneticfield dependence of the internal excited state of the polaron and the qubit in quantum dot with thickness. Journal of the Korean Physical Society, 70 (11), 956-961.

[42] Xin, W., \& Wang, G. S. (2018). Impurity, LO Phonon and Thickness Effects on the Transition of an Electron in a Gaussian Confinement Potential DQD with a Magnetic Field. Journal of Low Temperature Physics, 193 (1-2), 48-59.

[43] Kenfack, S. C., Fotué, A. J., Fobasso, M. F. C., Bawe Jr, G. N., \& Fai, L. C. (2017). Shannon entropy and decoherence of polaron in asymmetric polar semiconductor quantum wire. Superlattices and Microstructures, 111, 32-44. 